\title{
All-Optical Switching Based on Cross-Phase Modulation in Microstructure Fiber
}

\author{
Jay E. Sharping, Marco Fiorentino, Prem Kumar, and Robert S. Windeler
}

\begin{abstract}
In this letter, we demonstrate all-optical switching of picosecond pulses between the output ports of a microstructure-fiber-based polarization Sagnac interferometer. High contrast switching of 2.6-ps FWHM signal pulses due to cross-phase modulation induced by 4.9-ps pump pulses in a 5.8-m long microstructure fiber is achieved at wavelengths near 1550 and $780 \mathrm{~nm}$. The spectral and temporal behavior of the switching device are investigated and compared with numerical simulations based on coupled-wave theory.
\end{abstract}

Index Terms-Cross-phase modulation, optical fibers, optical switches, photonic crystal fiber.

$\mathbf{R}$ ECENT advances in fabrication and characterization of microstructure fibers (MFs) [1]-[3] have allowed researchers to propose and demonstrate a number of viable photonic applications using this material [4]-[6]. Microstructure fibers exhibiting single-mode behavior over a wide wavelength range can be fabricated, which have a relatively small core area, allowing one to take greater advantage of the weak $\chi^{(3)}$ nonlinearity of glass. By doing so, one reduces the pump power and fiber length requirements, the principal drawbacks of previously demonstrated fiber-based nonlinear-optic devices [7]-[15]. Furthermore, the MF design parameters permit greater control over the dispersion properties of the fiber, and hence, the MFs can be designed to favor certain pulse propagation effects or nonlinear interactions.

A number of research groups have investigated all-optical switching in fibers. Islam et al. [11] implemented an imbalanced nonlinear optical loop mirror wherein the amount of light transmitted by the loop was a function of the input pulse energy. Switching, in that case, was based on self-phase modulation (SPM). Blow et al. [12] also used a loop-mirror configuration, except that two wavelengths were used and switching was based on cross-phase modulation (XPM). The behavior of a variety of proposed switching configurations was investigated by Moores et al. [13], who gave special consideration to the temporal effects on switched pulses. More recently, Petropoulos et al. [14] reported using MF to implement a single wavelength regenerative switch, which operates on the basis of SPM combined with spectral filtering.

Manuscript received August 16, 2001; revised October 1, 2001. This research was supported in part by the U.S. Army Research Office under Grant DAAD19-00-1-0177 and Grant DAAD19-00-1-0469.

J. Sharping, M. Fiorentino, and P. Kumar are with the Center for Photonic Communication and Computing, ECE Department, Northwestern University, Evanston, IL 60208 USA.

R. S. Windeler is with Bell Laboratories, Lucent Technologies, Murray Hill, NJ 07974 USA.

Publisher Item Identifier S 1041-1135(02)00114-3.
In this letter, we report experimental demonstration of an all-optical switch, which operates on the basis of XPM in MF. Our apparatus exhibits several improvements over the previously demonstrated devices [7]-[15]; such as, it requires shorter fiber lengths, lower switching powers, and allows switching of weaker pulses. These experiments, thus, demonstrate the feasibility of using nonlinear optics in MF to perform essential functions in high-speed all-optical processing.

XPM can be understood by considering the evolution of two spectrally nonoverlapping pulses propagating through an optical fiber as governed by [16]

$$
\begin{aligned}
\frac{\partial A_{p}}{\partial z}+\frac{\alpha}{2} A_{p}+i \frac{\beta_{2}}{2} \frac{\partial^{2} A_{p}}{\partial^{2} T}= & i \gamma\left(\left|A_{p}\right|^{2}\right. \\
& \left.+2\left|A_{s}\right|^{2}\right) A_{p} \\
\frac{\partial A_{s}}{\partial z}+\frac{\alpha}{2} A_{s}+d \frac{\partial A_{s}}{\partial T}+i \frac{\beta_{2}}{2} \frac{\partial^{2} A_{s}}{\partial^{2} T}= & i \gamma\left(2\left|A_{p}\right|^{2}\right. \\
& \left.+\left|A_{s}\right|^{2}\right) A_{s} .
\end{aligned}
$$

Here, $A_{p(s)}$ represents the slowly varying envelope of the pump (signal) pulses at angular frequency $\omega_{p(s)}, \alpha$ and $\beta_{2}$ are the linear loss and the group-velocity dispersion (GVD) coefficients (assumed to be the same at both $\omega_{p}$ and $\omega_{s}$ ), respectively, $T=$ $t-z / v_{g p}$ is measured in a frame of reference moving with the pump pulses at group velocity $v_{g p}$, and $d=v_{g s}^{-1}-v_{g p}^{-1}$ parameterizes the group-velocity mismatch between the pump-andsignal pulses. The nonlinearity is included in the coefficient $\gamma=n_{2} \omega / A_{\text {eff }} c$ with $\omega_{p} \simeq \omega_{s}=\omega$. Equation (1) and (2) include the effects of linear loss, GVD, SPM, and XPM. We assume that four-wave mixing is not phase matched and can be ignored.

XPM can be used for optical switching by dividing the signal pulses equally on two arms of an interferometer and injecting the strong pump pulses only on one arm. Because the pump pulses co propagate with only one of the two signal pulses, there exists an XPM-induced phase difference $\phi_{\mathrm{NL}}=2 \gamma P_{p} L$ at the output of the interferometer, where $P_{p}$ is the peak power of the pump, and $L$ is the interaction length. By varying the intensity of the pump pulses, one can vary the magnitude of this phase difference. If a $\pi$-phase shift is achieved, one can switch the interference from destructive to constructive, or vice-versa.

The MF used in our experiments was $5.8 \mathrm{~m}$ long and was fabricated at Bell Labs, Lucent Technologies [2]. It consists of $\mathrm{a} \simeq 1.7-\mu \mathrm{m}$ diameter silica core surrounded by a hexagonal array of $\simeq 1.4 \mu \mathrm{m}$ diameter air voids. Asymmetry in the core results in a birefringent polarization-maintaining fiber. The attenuation coefficient $\alpha$ was measured to be $0.092 \pm 0.003 \mathrm{~m}^{-1}$, and $0.02 \pm 0.01 \mathrm{~m}^{-1}$ near 1550 and $780 \mathrm{~nm}$, respectively, by 


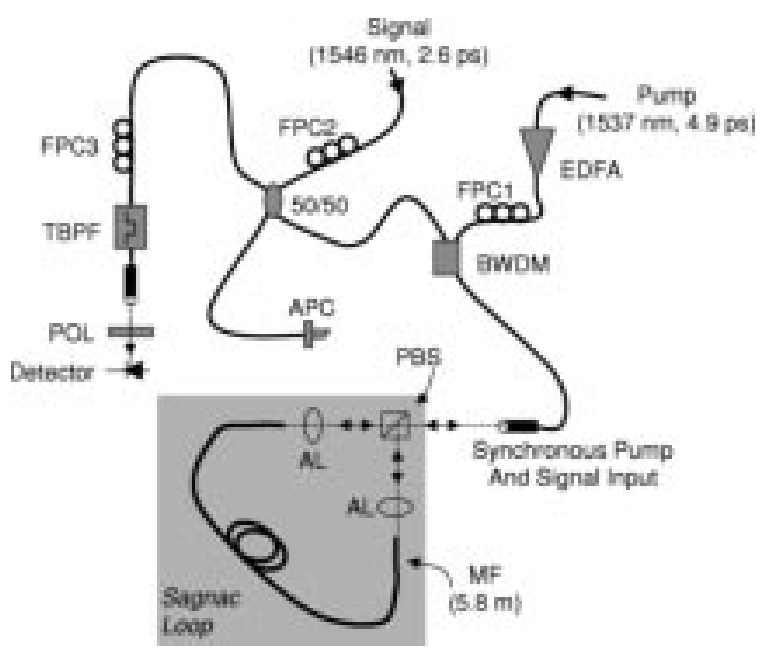

Fig. 1. Experimental setup used to demonstrate all-optical switching near 1550 nm. AL: Aspheric lens. APC: Angle polished connector. POL: polarizer.

comparing the insertion losses for two different MF lengths. The GVD coefficient $\beta_{2}$ was measured to be $-122 \pm 5 \mathrm{ps}^{2} / \mathrm{km}$ near $1550 \mathrm{~nm}$, and is negligible near $780 \mathrm{~nm}$ [2].

Fig. 1 shows the experimental setup that we used to observe switching near $1550 \mathrm{~nm}$ (we employed a similar apparatus using bulk-optic rather than fiber-optic components to conduct experiments near $780 \mathrm{~nm}$ ). We create synchronous pump-and-signal pulses by taking the $75-\mathrm{MHz}$ train of 150 -fs pulses centered at $1540 \mathrm{~nm}$ from a Ti:sapphire-pumped optical parametric oscillator system (Coherent, Inc., Models Mira 900 and Mira OPO), dispersing them with a grating and then spectrally filtering them to obtain two synchronous pulse trains with a tunable wavelength separation of 5-15 nm. This arrangement gives Gaussianshaped pump-and-signal pulses of $4.9 \pm 0.2 \mathrm{ps}$ and $2.6 \pm 0.2 \mathrm{ps}$ full-width at half-maximum (FWHM), respectively. The pump pulses are amplified by an erbium-doped fiber amplifier (EDFA) and combined with the signal pulses $(130 \mathrm{~mW}$ peak power) via a bandpass wavelength-division multiplexor (BWDM). The optical paths of the pump-and-signal pulses are matched so that they overlap temporally after the BWDM. The combined pulses are then injected into a Sagnac loop formed by a polarization beamsplitter cube (PBS) and a 5.8-m segment of the MF. Separate control of the polarization of the pump pulses (using FPC1) and the signal pulses (using FPC2) allows us to balance the signal in both directions through the Sagnac loop, while directing the pump in only one direction. By including a $90^{\circ}$ twist in the fiber orientation, the counterpropagating signal pulses and the pump pulses can be injected into the same polarization mode of the MF, which after one pass through the MF are directed back toward the source by the PBS. The returning signal and pump pulses are separated from each other as they pass through the BWDM in the opposite direction. The polarization of the returning signal pulses is adjusted with use of another fiber polarization controller FPC3. The signal pulses are then passed through a tunable bandpass filter (TBPF) to remove any remaining pump power $(<-15 \mathrm{~dB}$ that gets transmitted through the BWDM) and directed onto a polarizer for subsequent analysis. This analyzing polarizer can be aligned to detect either "port" of the switch, where the signal power increases as the

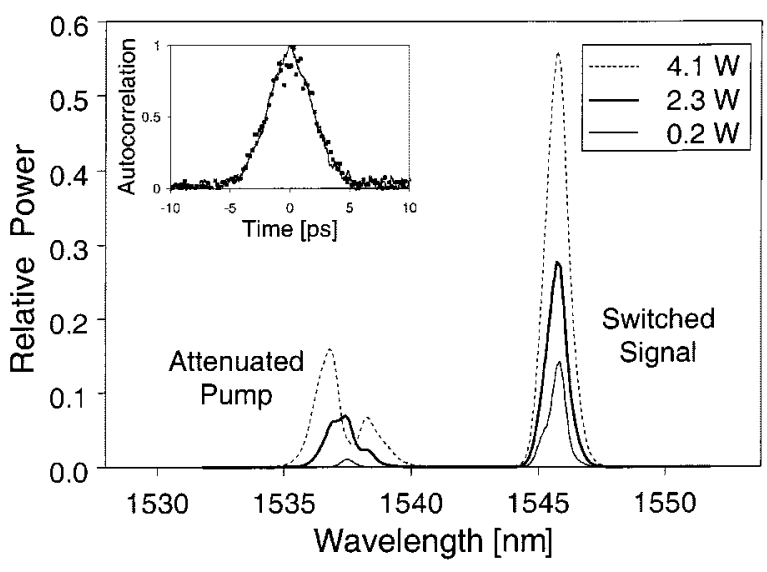

Fig. 2. Optical spectra showing increase in the switched signal power for pump peak powers of $0.2,2.3$, and $4.1 \mathrm{~W}$. Spectral broadening of the pump due to SPM also occurs as the pump power is increased. The inset shows autocorrelation traces of the input signal (line) and the switched signal (boxes) pulses.

pump power is increased, or the "port," where the signal power decreases as the pump power is increased.

In Fig. 2, we show the spectrum of the switched signal pulses with the TBPF removed, so that the spectrum of the pump pulses can also be observed. As the pump power is increased, larger XPM of the signal pulses by the pump pulses results in an increase in the switched signal power. Spectral broadening of the pump pulses due to SPM is also seen. The inset in Fig. 2 shows autocorrelation traces of the input and switched signal pulses. The shape and width of the switched signal pulses are nearly the same as those of the input pulses.

Measurements of the switched signal power versus pump peak power for wavelengths near $1550 \mathrm{~nm}$ are shown in Fig. 3(a). The data show that we were able to achieve slightly less than a $\pi$ nonlinear phase shift; our limitation being set by the power of the pump source. At wavelengths near 1550 $\mathrm{nm}, \mathrm{GVD}$ in the MF causes the pulses to walk off from each other within about $5 \mathrm{~m}$; so the use of a longer fiber would not result in a larger nonlinear phase shift. In addition, the interaction is limited by the effective fiber length [16], $L_{\mathrm{eff}}=\left(1-e^{-\alpha L}\right) / \alpha=4.5 \mathrm{~m}$ in our case. For comparison, in Fig. 3(b), we show the results of a similar experiment performed at wavelengths near $780 \mathrm{~nm}$. In this case, we were able to observe a larger nonlinear phase shift (about $2 \pi$ ) because the nonlinear coefficient $\gamma$ scales inversely with wavelength, $L_{\text {eff }}$ is larger, and the GVD coefficient is negligible near 780 $\mathrm{nm}$. Reflections of the input signal pulses from the uncoated tips of the MF introduce a background contribution to the average power measurements, which depending on the MF coupling efficiency, can amount to as much as $20 \%$ of the average switched power. This background could be removed by anti reflection coating of the fiber tips. For the data shown in Fig. 3(a), we separated the switched pulses from these reflected pulses by making time-resolved measurements using a fast photodiode.

The theoretical curves (solid) that accompany the data shown in Fig. 3, were generated by numerically solving (1) and (2) for the pump-and-signal pulses using a third-order Runge-Kutta split-step Fourier method. No fitting parameters were used in these simulations. The constants used in the simulation were the 


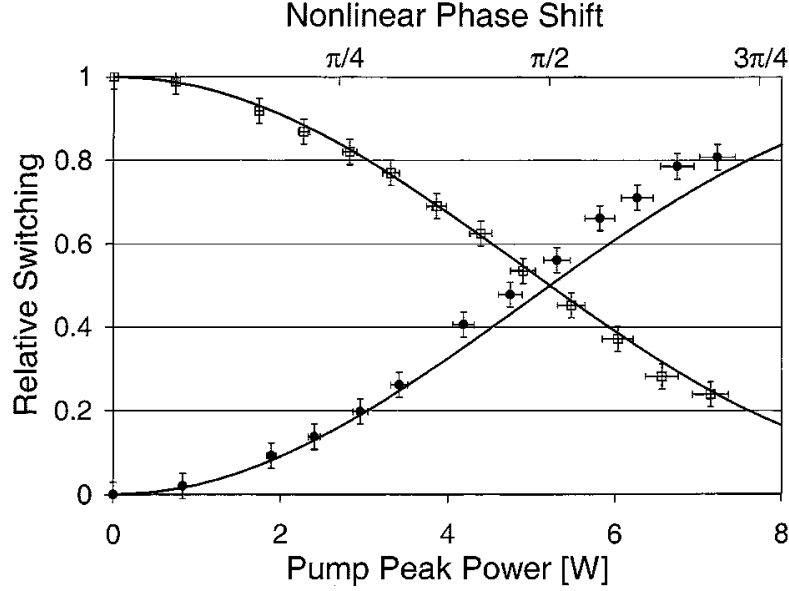

(a)

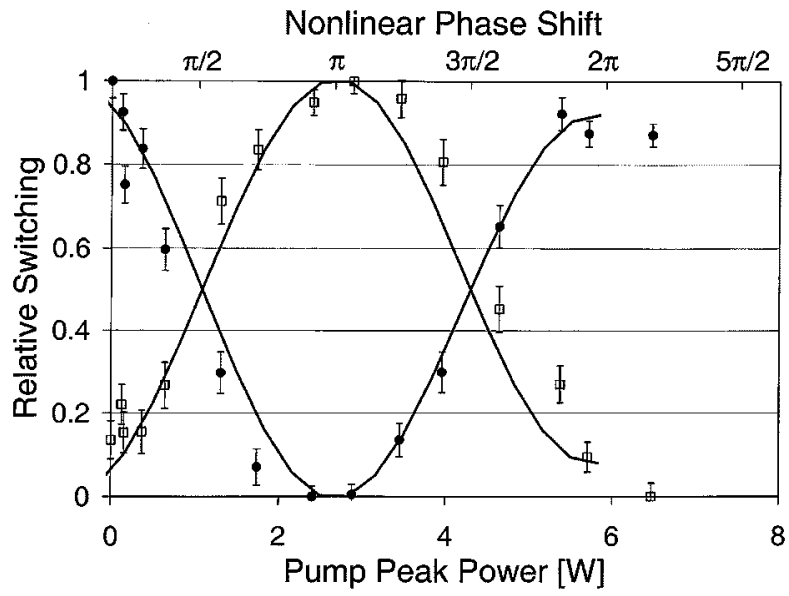

(b)

Fig. 3. Switching curves (open boxes and filled circles), showing the relative power measured in each port of the switch versus the pump peak power, for experiments conducted near (a) $1550 \mathrm{~nm}$ and (b) $780 \mathrm{~nm}$. The curves accompanying the data are generated from numerical solutions of (1) and (2).

effective mode area $\mathrm{A}_{\text {eff }}$, which was calculated from the core area, and the measured values of the fiber length, $\alpha, \beta_{2}$, and the FWHM of the pump-and-signal pulses. Since the core of this MF is made of pure silica, the nonlinear coefficient $\gamma$ was calculated using $n_{2}=2.8 \times 10^{-20} \mathrm{~m}^{2} / \mathrm{W}[16]$.

The pump peak power required for $50 \%$ switching at wavelengths near $1550 \mathrm{~nm}$ is $5.2 \mathrm{~W}$, which is comparable to the value of $6.6 \mathrm{~W}$ achieved by Kanter et al. [17], wherein a periodically poled lithium-niobate waveguide was used as the nonlinear medium. While full switching at $1550 \mathrm{~nm}$ was not possible with our pump source, the theory predicts a switching contrast greater than $93 \%$ at peak pump powers of about $10 \mathrm{~W}$ (corresponding to about $50 \mathrm{pJ}$ of switching energy). Such high-contrast switching can be seen in the data taken at $780 \mathrm{~nm}$ [Fig. 3(b)].

One criterion for evaluating all-optical switching devices is the pump peak power times the fiber length $(P L)$ for complete switching, where a smaller value indicates more efficient operation. Our device exhibits $P L \simeq 60 \mathrm{~m} \cdot \mathrm{W}$, whereas for the best previous result $P L \simeq 360 \mathrm{~m} \cdot \mathrm{W}$ [15]. The only other known MF-based switch [14] obtained $P L \simeq 130 \mathrm{~m} \cdot \mathrm{W}$. While our demonstration employed an elaborate optical parametric oscillator system, the use of commercially available electro-absorption modulators and high-power EDFAs for creating the pump pulses would give the same peak powers, resulting in similar switching performance.

In conclusion, we have demonstrated an all-optical switch that operates on the basis of XPM in MF. The spectral and temporal behavior as well as the pump-power dependence of the signal pulses were experimentally studied and are shown to be consistent with the theory.

\section{ACKNOWLEDGMENT}

The authors gratefully acknowledge the assistance of G. Kanter and P. Limpiti.

\section{REFERENCES}

[1] J. C. Knight, T. A. Birks, P. St. J. Russell, and D. M. Atkin, "All-silica single-mode optical fiber with photonic crystal cladding," Opt. Lett., vol. 21, pp. 1547-1549, Oct. 1996.

[2] J. K. Ranka, R. S. Windeler, and A. J. Stentz, "Visible continuum generation in air-silica microstructure optical fibers with anomalous dispersion at $800 \mathrm{~nm}$," Opt. Lett., vol. 25, pp. 25-27, Jan. 2000.

[3]

[4] N. G. R. Broderick, T. M. Monro, P. J. Bennett, and D. J. Richardson, "Nonlinearity in holey optical fibers: Measurement and future opportunities," Opt. Lett., vol. , 24, pp. 1395-1397, Oct. 1999.

[5] J. E. Sharping, M. Fiorentino, A. Coker, P. Kumar, and R. S. Windeler, "Four-wave mixing in microstructure fiber," Opt. Lett., vol. 26, pp. 1048-1050, July 2001.

[6] X. Liu, C. Xu, W. H. Knox, J. K. Chandalia, B. J. Eggleton, S. G. Kosinski, and R. S. Windeler, "Soliton self-frequency shift in a short tapered air-silica microstructure fiber," Opt. Lett., vol. 26, pp. 358-360, Mar. 2001.

[7] D. K. Serkland and P. Kumar, "Tunable fiber-optic parametric oscillator," Opt. Lett., vol. 24, pp. 92-94, Jan. 1999.

[8] Y. Su, L. Wang, A. Agarwal, and P. Kumar, "Wavelength-tunable alloptical clock recovery using a fiber-optic parametric oscillator," Opt. Commun., vol. 184, pp. 151-156, Oct. 2000.

[9] - "Simultaneous 3R regeneration and wavelength conversion using a fiber-parametric limiting amplifier," in Tech. Dig. Optical Fiber Communication Conf., Anaheim, CA, Mar. 17-22, 2001, paper MG4-1.

[10] L. Wang, Y. Su, A. Agarwal, and P. Kumar, "An all-optical picosecond-pulse packet buffer based on four-wave mixing loading and intracavity soliton control," in Postdeadline Papers: Conference on Lasers and Electro-Optics ., San Francisco, CA, 2000, paper CPD20.

[11] M. N. Islam, E. R. Sunderman, R. H. Stolen, W. Pleibel, and J. R. Simpson, "Soliton switching in a fiber nonlinear loop mirror," Opt. Lett., vol. 14, pp. 811-813, Aug. 1989.

[12] K. J. Blow, N. J. Doran, B. K. Nayar, and B. P. Nelson, "Two-wavelength operation of the nonlinear fiber loop mirror," Opt. Lett., vol. 15 , pp. 248-250, Feb. 1990.

[13] J. D. Moores, K. Bergman, H. A. Haus, and E. P. Ippen, "Optical switching using fiber ring reflectors," J. Opt. Soc. Amer. B., vol. 8, pp. 594-601, Mar. 1991.

[14] P. Petropoulos, T. M. Monro, W. Belardi, K. Furusawa, J. H. Lee, and D. J. Richardson, "A highly nonlinear holey fiber and its application in a regenerative optical switch," in Tech. Dig. Optical Fiber Communication Conf., Anaheim, CA, Mar. 17-22, 2001, paper TuC3.

[15] M. Jinno and T. Matsumoto, "Ultrafast, low power, and highly stable all-optical switching in an all polarization maintaining fiber Sagnac interferometer," IEEE Photon. Technol. Lett., vol. 2, pp. 349-351, May 1990.

[16] G. P. Agrawal, Nonlinear Fiber Optics, second ed. New York: Academic, 1995

[17] G. S. Kanter, P. Kumar, K. R. Parameswaran, and M. M. Fejer, "Wavelength-selective pulsed all-optical switching based on cascaded secondorder nonlinearity in a periodically poled lithium-niobate waveguide," IEEE Photon. Technol. Lett ., vol. 13, pp. 341-343, Apr. 2001. 